\title{
Provenance of classical Hamiltonian time crystals
}

\author{
Anton Alekseev, ${ }^{a}$ Jin Dai ${ }^{b}$ and Antti J. Niemi ${ }^{b, c, 1}$ \\ ${ }^{a}$ Section of Mathematics, Université de Gèneve, \\ 2-4 rue du Lièvre, Case postale 641211 Genève 4, Switzerland \\ ${ }^{b}$ Nordita, Stockholm University, \\ Roslagstullsbacken 23, SE-106 91 Stockholm, Sweden \\ ${ }^{c}$ Laboratoire de Mathematiques et Physique Theorique CNRS UMR 7350, \\ Fédération Denis Poisson, Université de Tours, \\ Parc de Grandmont, F37200, Tours, France \\ E-mail: Anton.Alekseev@unige.ch, jin.dai@fysik.su.se, \\ Antti.Niemi@su.se
}

AbStract: Classical Hamiltonian systems with conserved charges and those with constraints often describe dynamics on a pre-symplectic manifold. Here we show that a presymplectic manifold is also the proper stage to describe autonomous energy conserving Hamiltonian time crystals. We explain how the occurrence of a time crystal relates to the wider concept of spontaneously broken symmetries; in the case of a time crystal, the symmetry breaking takes place in a dynamical context. We then analyze in detail two examples of timecrystalline Hamiltonian dynamics. The first example is a piecewise linear closed string, with dynamics determined by a Lie-Poisson bracket and Hamiltonian that relates to membrane stability. We explain how the Lie-Poisson brackets descents to a timecrystalline pre-symplectic bracket, and we show that the Hamiltonian dynamics supports two phases; in one phase we have a time crystal and in the other phase time crystals are absent. The second example is a discrete one dimensional model of a Hamiltonian chain. It is obtained by a reduction from the Q-ball Lagrangian that describes time dependent nontopological solitons. We show that a time crystal appears as a minimum energy domain wall configuration, along the chain.

KEYwords: Differential and Algebraic Geometry, Field Theories in Lower Dimensions

ARXIV EPRINT: 2002.07023

\footnotetext{
${ }^{1}$ Corresponding author.
} 


\section{Contents}

1 Introduction 1

2 Hamiltonian time crystals 2

3 Example 1: time crystals and closed discrete strings 5

3.1 Three-vertex model 8

$\begin{array}{ll}3.2 & \text { Four-vertex model }\end{array}$

4 Example 2: time crystals from a reduction of the Q-ball Lagrangian 11

5 Conclusions 17

\section{Introduction}

A classical Hamiltonian time crystal is an autonomous, time periodic solution of Hamilton's equation that is simultaneously a local minimum of the energy. Accordingly a time crystal spontaneously breaks the continuous time translation symmetry into discrete time translations [1-4]. (For a review see e.g. [5, 6].) This symmetry breakdown is analogous to the way how an ordinary crystalline material breaks the group of continuous spatial translations into a discrete Bravais lattice.

There are numerous examples of periodically driven nonlinear oscillators, and many other kind of open and non-equilibrium physical systems that display periodic time dependence. However, these examples do not qualify as time crystals: the breakdown of time translation symmetry is explicit and reflects verbatim the properties of the external forces and ambient interactions. Veritable timecrystalline dynamics [5-13] has also been experimentally observed [14-21], originally in the context of certain externally driven non-equilibrium spin chains $[14,15]$.

In the known examples a spin chain is subjected to an extrinsic, periodic driving force. This prompts the spin chain to respond in a time periodic fashion, but now the response comes with an intrinsic periodicity that is different from the period of the drive.

Whenever an energy conserving, isolated Hamiltonian system has been found to display time periodic dynamics, such as in the case of a harmonic oscillator or the Kepler problem, the motion can always be removed in a natural fashion, by a continuous deformation of the system towards its lowest available energy state. Indeed, it is widely thought that in the case of a closed autonomous Hamiltonian system, any kind of timecrystalline dynamics is excluded [22, 23]. This conclusion is grounded on the structure of the textbook Hamilton's equation

$$
\begin{aligned}
& \frac{d q^{a}}{d t}=\left\{q^{a}, H\right\}=\frac{\partial H}{\partial p^{a}} \\
& \frac{d p^{a}}{d t}=\left\{p^{a}, H\right\}=-\frac{\partial H}{\partial q^{a}}
\end{aligned}
$$


On a compact, closed manifold a minimum energy configuration is also a critical point of the Hamiltonian $H$. Thus, at the energy minimum the right hand sides of (1.1) vanish, which implies that the left hand sides must vanish as well. As a consequence a trajectory that is a minimum of $H$ must be time independent and one concludes that Hamilton's equation (1.1) can not support any timecrystalline solution.

However, we have found that there is a systematic way to evade these Hamiltonian No-Go arguments. This is exemplified by the following simple scenario: suppose that $h=h(x, y)$ is a smooth real valued function defined on a compact closed manifold. For example we may take the manifold to be the Riemann sphere i.e. the plane $\mathbb{R}^{2}$ plus a point at infinity. Assume that the function has only non-degenerate maxima and minima on the sphere, these are located at critical points $\partial_{x} h=\partial_{y} h=0$. Now consider another smooth real valued function $g(x, y)$ and introduce a condition such as

$$
g(x, y)=c
$$

Whenever $c$ is a regular value of $g$ the solution defines a curve on the sphere. Generically, for a given value of $c$ we do not expect that the curve passes through any of the critical points of $h(x, y)$. Thus, for a generic given $c$ the minimal values that $h(x, y)$ attains along the curve $g(x, y)=c$ are not among its critical points on the sphere. It is even possible that this is the case for all those values of $c$ that are allowed by the structure of $g(x, y)$

Recently, explicit examples of classical Hamiltonian time crystals have been presented [24]. The examples go around the No-Go arguments in the manner that we have outlined: they are Hamiltonian systems with conserved quantities, but the numerical values of the conserved charges are constrained. This causes the Hamiltonian system to become timecrystalline, with a spontaneously broken time translation symmetry. In particular, the ensuing phase spaces are not symplectic manifolds. Instead, the timecrystalline dynamics takes place on a more general pre-symplectic manifold [25] where the No-Go arguments are circumvented.

We start and trace the provenance of Hamiltonian timecrystalline dynamics to the geometry of the phase space. We explain in a general context why pre-symplectic geometry is necessary for the emergence of timecrystalline dynamics, in the case of Hamilton's equation with conserved charges. We then construct in detail two examples of Hamiltonian time crystals, both examples have their origin and motivation in familiar field theoretical models.

\section{Hamiltonian time crystals}

Hamilton's equation describes energy conserving dynamics on a $2 N$ dimensional symplectic manifold $\mathcal{M}$; for background on geometric mechanics see e.g. [25]. The manifold is equipped with a closed and non-degenerate two-form

$$
\begin{aligned}
\Omega & =\Omega_{a b} d \phi^{a} \wedge d \phi^{b} \\
d \Omega & =0
\end{aligned}
$$

where $\phi^{a}(a=1, \ldots, 2 N)$ are generic local coordinates on $\mathcal{M}$. For pedagogy we prefer to use local coordinate representation even though all our relations are coordinate independent. 
Hamilton's equation is

$$
\Omega_{a b} \frac{d \phi^{b}}{d t}=\partial_{a} H
$$

where the Hamiltonian $H(\phi)$ models the mechanical free energy, it is assumed to be a smooth real valued function on $\mathcal{M}$. The solutions $\phi^{a}(t)$ are non-intersecting trajectories on $\mathcal{M}$, they are uniquely specified by the initial values $\phi^{a}(0)$. The inverse of the matrix $\Omega_{a b}$ defines the Poisson brackets

$$
\left\{\phi^{a}, \phi^{b}\right\}=\Omega^{a b}(\phi)
$$

so that (2.2) becomes

$$
\frac{d \phi^{a}}{d t}=\left\{\phi^{a}, H\right\}=\Omega^{a b} \partial_{b} H
$$

Darboux theorem ensures that there is a local coordinate transformation on the manifold $\mathcal{M}$ such that the $\phi^{a}$ become the $\left(p^{a}, q^{a}\right)$ that are equipped with their canonical Poisson brackets, and Hamilton's equation (2.4) acquires the familiar form (1.1).

We define a time crystal to be a minimum energy solution of Hamilton's equation (2.2), (2.4) with a non-trivial $t$-dependence that we assume is periodic $\phi^{a}(t+T)=\phi^{a}(t)$.

We search for time crystals in Hamiltonian systems with symmetries. Noether's theorem states that a symmetry gives rise to a conservation law and we denote the pertinent conserved charges $G_{i}(\phi)(i=1, \ldots, n \leq N)$. Their Poisson brackets with the Hamiltonian $H(\phi)$ vanish,

$$
\left\{H, G_{i}\right\}=\frac{d G_{i}}{d t}=0
$$

Furthermore, the Poisson brackets of the $G_{i}$ closes with a Lie algebra structure

$$
\left\{G_{i}, G_{j}\right\}=f_{i j}^{k} G_{k}
$$

of the symmetry group.

We assign numerical values $G_{i}(\phi(0))=g_{i}$ to the conserved charges. The $g_{i}$ are regular values of the $G_{i}(\phi)$, they are determined in terms of the initial conditions $\phi^{a}(0)$ of Hamilton's equation. The level sets $G_{i}(\phi(0))=g_{i}$ foliate the symplectic manifold $\mathcal{M}$, and we specify them by conditions

$$
\mathcal{G}_{i}^{g}(\phi)=G_{i}(\phi)-g_{i}=0
$$

For each $g_{i}$ the condition (2.6) specifies a submanifold of $\mathcal{M}$ that we denote by $\mathcal{M}_{g}$.

The Poisson brackets of (2.6) are

$$
\left\{\mathcal{G}_{i}^{g}, \mathcal{G}_{j}^{g}\right\}=f_{i j}{ }^{k} \mathcal{G}_{k}^{g}+f_{i j}{ }^{k} g_{k}
$$

where the matrix

$$
\gamma_{i j}(g)=f_{i j}{ }^{k} g_{k}
$$

has a rank $s \leq n$ that in general depends on the values $g_{i}$. Following Dirac [26] we regard (2.6) as a combination of $(n-s)$ first class conditions and $s$ second class conditions. 
The first class conditions correspond to those combinations of $G_{i}(\phi)$ that define the kernel of $\gamma_{i j}(g)$ and the second class conditions span the image of $\gamma_{i j}(g)$.

For each regular values $g_{i}$ in (2.6) we restrict the non-degenerate symplectic twoform (2.1) to the corresponding submanifold $\mathcal{M}_{g}$

$$
\Omega_{\left.\right|_{\mathcal{M}_{g}}} \equiv \omega^{g}=\omega^{g}(\phi)_{a b} d \phi^{a} \wedge d \phi^{b}
$$

The two-form $\omega^{g}$ is closed but in general the matrix $\omega_{a b}^{g}$ is degenerate with a $(n-s)$ dimensional kernel. Accordingly, whenever $n-s \neq 0$ the submanifold $\mathcal{M}_{g}$ that we equip with the closed two-form (2.9), is not a symplectic manifold but a pre-symplectic manifold. That is, simply a manifold with a closed two-form.

In the following we assume that the physical circumstances are such that for all regular values $g_{i}$ of interest in (2.6) we have $n-s \neq 0$ so that the corresponding manifolds $\mathcal{M}_{g}$ are pre-symplectic. Since the No-Go arguments [22, 23] assume that Hamiltonian dynamics takes place on a symplectic manifold, those arguments no longer apply. Thus, in the case of a pre-symplectic manifold $\mathcal{M}_{g}$ the existence of a timecrystalline solution to Hamilton's equation can not be excluded.

We note that there are many Hamiltonian dynamical systems with conserved charges. However, not all of them can support a time crystal. The existence of a time crystal depends on the way how the regular values $g_{i}$ in (2.6) are distributed into subsets $\left\{g_{i}\right\} \subset \mathbb{R}^{n}$. In general there can be multiple disconnected subsets, and each connected component pertains to a specific physical scenario. A necessary condition for a given subset $\left\{g_{i}\right\}$ to support timecrystalline dynamics is, that this subset can not be path connected to a stationary point of the Hamiltonian with a lower energy, in a manner that is dictated by the specifics of the physical scenario. ${ }^{1}$ The concrete examples that we present in the sequel, elucidate this point.

To reveal the actual presence of a time crystal we need to locate the minimum value of the Hamiltonian $H(\phi)$. Since the $G_{i}(\phi)$ are conserved it suffices to restrict the search of the minimum to the submanifolds $\mathcal{M}_{g}$ of interest. For this we account for (2.6) in terms of Lagrange multipliers $\lambda^{i}$ and extend the Hamiltonian $H(\phi)$ into

$$
H \rightarrow H_{\lambda}=H+\lambda^{i}\left(G_{i}-g_{i}\right)
$$

The Lagrange multiplier theorem [25] states that on a given submanifold (2.6) the minimum value $\phi_{\mathrm{cr}}^{a}$ of the Hamiltonian $H(\phi)$ coincides with a critical point $\left(\phi_{\mathrm{cr}}^{a}, \lambda_{\mathrm{cr}}^{i}\right)$ of the Hamiltonian function $H_{\lambda}(\phi)$. Thus the minimum value of $H(\phi)$ on $\mathcal{M}_{g}$ is obtained as a solution of

$$
\left\{\begin{array}{l}
{\frac{\partial H}{\partial \phi^{a}}{ }_{\mid \phi_{\mathrm{cr}}}}=-\lambda_{\mathrm{cr}}^{i} \frac{\partial G_{i}}{\partial \phi^{a}}{ }_{\mid \phi_{\mathrm{cr}}} \\
G_{i}\left(\phi_{\mathrm{cr}}\right)=g_{i}
\end{array}\right.
$$

Accordingly, we search for a time crystal using the following steps:

\footnotetext{
${ }^{1}$ This excludes examples such as the Kepler problem where the angular momentum can continuously decrease until it vanishes, and the harmonic oscillator where we can continuously diminishing the energy.
} 
- From the equations (2.11) we first solve for the minima $\phi_{\mathrm{cr}}^{a}$ of $H(\phi)$ on the submanifolds $\mathcal{M}_{g}$ of interest. Here the set of $\mathcal{M}_{g}$ includes all the level surfaces of the conserved charges that correspond to the physical scenario.

- We then continue and solve (2.11) for the corresponding values $\lambda_{\mathrm{cr}}^{i}$ in terms of $\phi_{\mathrm{cr}}^{a}$.

Whenever $\lambda_{\mathrm{cr}}^{i}\left(\phi_{\mathrm{cr}}\right) \neq 0$ the minimum energy solution $\phi_{\mathrm{cr}}^{a}$ can be employed as an initial value to a timecrystalline solution of Hamilton's equation (2.4). In the case of a time crystal, Hamilton's equation then becomes

$$
\begin{aligned}
& \frac{d \phi^{a}}{d t}=-\Omega^{a b} \lambda_{\mathrm{cr}}^{i} \frac{\partial G_{i}}{\partial \phi^{b}} \neq 0 \\
& \phi^{a}(0)=\phi_{\mathrm{cr}}^{a}
\end{aligned}
$$

Note that the Lagrange multipliers $\lambda_{\mathrm{cr}}^{i}$ are $t$-independent, their values for all $t$ are determined by (2.11) in terms of the initial values $\phi_{\mathrm{cr}}^{a}$. This follows immediately, since both $H(\phi)$ and $G_{i}(\phi)$ are by construction $t$-independent along any Hamiltonian trajectory.

The emergence of a time crystal is a manifestation of the general phenomenon of spontaneous symmetry breaking: a time crystal describes a time dependent minimum energy symmetry transformation of the Hamiltonian $H(\phi)$ that is generated by the linear combination

$$
G_{\lambda}(\phi) \equiv \lambda_{\mathrm{cr}}^{i} G_{i}(\phi)
$$

Thus a time crystal breaks the full symmetry group of conserved charges (2.5) into an abelian U(1) symmetry transformation. We remark that in general the ensuing motion (2.12) is quasi-periodic, but here we assume it to be periodic $\phi^{a}(t+T)=\phi^{a}(t)$ and the period $T$ is specified by $\lambda_{\mathrm{cr}}^{i}\left(\phi_{\mathrm{cr}}\right)$.

It is apparent that the present remarks are merely an invitation for a judicious mathematical investigation, and we propose that the methods of equivariant Morse theory [27-30] can be adopted to develop a mathematical framework for understanding Hamiltonian time crystals; we plan to return to this in a future research and we now proceed to exemplify our general remarks by a detailed analysis of two examples where timecrystalline dynamics appears in a familiar physical context.

\section{Example 1: time crystals and closed discrete strings}

In our first example we follow [24] and analyze timecrystalline dynamics in the context of a Hamiltonian system with time evolution determined by a Lie-Poisson bracket [25]. The Hamiltonian function we use has been originally introduced in [31-33], in connection of membrane stability analysis.

We start by explaining how the Lie-Poisson structure of [24] fits in our general framework: a Lie-Poisson bracket commonly describes the way how a Poisson manifold, i.e. a manifold that is equipped with a Poisson bracket, becomes foliated into symplectic leaves. Each leaf is a symplectic manifold, it supports Hamiltonian dynamics that is restrained on the given leaf. 
We consider a four dimensional phase space with Darboux coordinates $\left(q_{1}, q_{2}, p_{1}, p_{2}\right)$ that we combine into complex coordinates

$$
\begin{aligned}
& z_{1}=\frac{1}{2}\left(p_{i}+i q_{1}\right) / 2 \\
& z_{2}=\frac{1}{2}\left(p_{2}+i q_{2}\right) / 2
\end{aligned}
$$

with Poisson brackets

$$
\left\{z_{i}, z_{j}^{\star}\right\}=i \delta_{i j} \quad \& \quad\left\{z_{i}, z_{j}\right\}=\left\{z_{i}^{\star}, z_{j}^{\star}\right\}=0
$$

We introduce the Pauli matrices

$$
\sigma^{1}=\left(\begin{array}{ll}
0 & 1 \\
1 & 0
\end{array}\right) \quad \sigma^{2}=\left(\begin{array}{cc}
0 & -i \\
i & 0
\end{array}\right) \quad \sigma^{3}=\left(\begin{array}{cc}
1 & 0 \\
0 & -1
\end{array}\right)
$$

to define a vector with three real components

$$
n^{a}=-\frac{1}{2}\left(z_{1}^{\star}, z_{2}^{\star}\right) \sigma^{a}\left(\begin{array}{c}
z_{1} \\
z_{2}
\end{array}\right)
$$

and so that

$$
\mathbf{n} \cdot \mathbf{n}=\frac{1}{4}\left(\left|z_{1}\right|^{2}+\left|z_{2}\right|^{2}\right)^{2}=r^{4}
$$

The $n^{a}$ obey the SU(2) Lie-Poisson brackets

$$
\left\{n^{a}, n^{b}\right\}=\epsilon^{a b c} n^{c}
$$

and the length (3.4) is preserved by the action of the $n^{a}$

$$
\left\{n^{a}, \mathbf{n} \cdot \mathbf{n}\right\}=0
$$

The phase space (3.1) is a model space of $\mathrm{SU}(2)$ representations, and different values of $r$ correspond to different representations. We identify the symplectic structures of the $\mathrm{SU}(2)$ coadjoint orbits in terms of local coordinates

$$
\left(\begin{array}{l}
z_{1} \\
z_{2}
\end{array}\right)=r\left(\begin{array}{c}
\cos \frac{\theta}{2} e^{i(\chi+\phi) / 2} \\
\sin \frac{\theta}{2} e^{i(\chi-\phi) / 2}
\end{array}\right)
$$

This yields

$$
\mathbf{n}=\left(\begin{array}{l}
n_{1} \\
n_{2} \\
n_{3}
\end{array}\right)=r^{2}\left(\begin{array}{c}
\cos \phi \sin \theta \\
\sin \phi \sin \theta \\
\cos \theta
\end{array}\right)
$$

and for the symplectic two-form of (3.2) we get

$$
\Omega=i d z_{1}^{\star} \wedge d z_{1}+i d z_{2}^{\star} \wedge d z_{2}=r d \chi \wedge d r+r \cos \theta d \phi \wedge d r+\frac{r^{2}}{2} d \phi \wedge d \cos \theta
$$


from which we read the following Poisson brackets

$$
\begin{aligned}
\{r, \chi\} & =\frac{1}{r} \\
\{\cos \theta, \chi\} & =-\frac{2}{r^{2}} \cos \theta \\
\{\cos \theta, \phi\} & =\frac{2}{r^{2}} \\
\{r, \cos \theta\}=\{r, \phi\} & =\{\chi, \phi\}=0
\end{aligned}
$$

The coordinates in the r.h.s. of (3.7) are simply spherical coordinates on $\mathbb{R}^{4}$, the $(\theta, \phi, \chi)$ are angular coordinates that describe the spheres $\mathbb{S}^{3}$ that foliate $\mathbb{R}^{4}$ with radii $r^{2}$. The Hopf map $\mathbb{S}^{3} \rightarrow \mathbb{S}^{2}$ identifies $(\theta, \phi)$ as the latitude and longitude angles of a two-sphere, and $\chi$ is the coordinate of the remaining $\mathbb{S}^{1}$. The two-spheres are the orbits of $\mathrm{SU}(2)$ representations, and each two-sphere is equipped with a symplectic two-form that corresponds to the Poisson bracket (3.11), with $\cos \theta$ and $\phi$ a canonical pair.

We proceed to describe a physical scenario where $N$ such vectors $\mathbf{n}_{i}$ appear as dynamical degrees of freedom, each equipped with its own Lie-Poisson bracket (3.5) [24]. For this we interpret the vectors as links that connect the $N+1$ vertices $\mathbf{x}_{i}$ of a piecewise linear polygonal string in $\mathbb{R}^{3}$

$$
\mathbf{n}_{i}=\mathbf{x}_{i+1}-\mathbf{x}_{i}
$$

Since

$$
\left\{\mathbf{n}_{i}, \mathbf{n}_{k} \cdot \mathbf{n}_{k}\right\}=0 \quad \text { for all } i, k
$$

the lengths of the links remain intact during time evolution, whenever the Hamiltonian function depends only on the vectors $\mathbf{n}_{i}$ which we assume to be the case. For convenience we set all the link lengths to have the equal value $\left|\mathbf{n}_{i}\right|=1$.

With $H(\mathbf{n})$ a Hamiltonian function, the Lie-Poisson bracket (3.5) yields the following Hamilton's equation

$$
\frac{\partial \mathbf{n}_{i}}{\partial t}=\left\{\mathbf{n}_{i}, H(\mathbf{n})\right\}=-\mathbf{n}_{i} \times \frac{\partial H}{\partial \mathbf{n}_{i}}
$$

and we proceed to reveal its timecrystalline dynamics.

To introduce the conserved charges (2.6) we consider the vector

$$
\mathbf{G}=\sum_{i=1}^{N} \mathbf{n}_{i}
$$

Its components obey the Poisson brackets

$$
\left\{G^{a}, G^{b}\right\}=\epsilon^{a b c} G^{c}
$$

and we choose a Hamiltonian such that

$$
\{H(\mathbf{n}), \mathbf{G}\}=0
$$

The $\mathbf{G}$ are the conserved charges of interest. The following is then an example of the condition (2.6): we define the manifolds $\mathcal{M}_{g}$ by

$$
G^{a}=g^{a} \equiv\left(x_{N+1}^{a}-x_{1}^{a}\right)
$$


and the matrix $\gamma_{i j}$ in (2.8) is

$$
\gamma_{a b} \sim \epsilon^{a b c}\left(x_{N+1}^{c}-x_{1}^{c}\right)
$$

We now specify the physical scenario that is of interest to us: we describe the dynamics of a closed string, and for this we set $\mathbf{x}_{N+1}=\mathbf{x}_{1}$. Thus (2.6) becomes

$$
\mathbf{G}=\sum_{i=1}^{N} \mathbf{n}_{i}=0
$$

The entire algebra (2.7) of the conserved charges is first class.

In line with our general formalism we introduce the Lagrange multiplier $\boldsymbol{\lambda}$ so that the Hamiltonian (2.10) in the present case is

$$
H_{\boldsymbol{\lambda}}=H(\mathbf{n})+\boldsymbol{\lambda} \cdot \mathbf{G}
$$

For a time crystal, the equation (2.12) yields us the following

$$
\frac{\partial \mathbf{n}_{i}}{\partial t}=-\boldsymbol{\lambda}_{\mathrm{cr}} \times \mathbf{n}_{i}
$$

where the Lagrange multiplier $\boldsymbol{\lambda}_{\mathrm{cr}}$ is evaluated at the minimum value of the Hamiltonian,

$$
\boldsymbol{\lambda}_{\mathrm{cr}}=-\frac{\partial H}{\partial \mathbf{n}_{i} \mid \mathbf{n}_{\min }}
$$

If a solution with $\boldsymbol{\lambda}_{\mathrm{cr}} \neq 0$ exist we have a timecrystalline closed string that rotates as a rigid body. The rotation axis points in the direction of $\boldsymbol{\lambda}_{\mathrm{cr}}$ and the magnitude of the angular velocity is given by the length $\left|\boldsymbol{\lambda}_{\mathrm{cr}}\right|$. Note that the r.h.s. involves the index $i=1, \ldots, N$ that is absent in the l.h.s. Thus (3.20) is a very stiff condition on the shape of the timecrystalline closed string.

We proceed to analyze in detail two such timecrystalline closed strings, with $N=3$ and $N=4$ vertices. For the Hamiltonian function, we follow [31-33] and select

$$
H=H_{1}+c H_{2}=\sum_{i=1}^{N}\left|\mathbf{n}_{i} \times \mathbf{n}_{i+1}\right|^{2}+c \sum_{i=1}^{N} \mathbf{n}_{i} \cdot\left(\mathbf{n}_{i+1} \times \mathbf{n}_{i+2}\right) \quad \text { with } \quad \mathbf{n}_{N+i}=\mathbf{n}_{i}
$$

\subsection{Three-vertex model}

For $N=3$ the closed string constraint (3.15) states that the variables $\mathbf{x}_{1}, \mathbf{x}_{2}, \mathbf{x}_{3}$ are the vertices of an equilateral triangle in $\mathbb{R}^{3}$. We can take $\mathbf{x}_{1}, \mathbf{x}_{2}, \mathbf{x}_{3}$ to lie on the $x y$-plane, with sites $\left|\mathbf{x}_{i+1}-\mathbf{x}_{i}\right|=1$. With the initial choice

$$
\begin{aligned}
& \mathbf{n}_{1}=(1,0,0) \\
& \mathbf{n}_{2}=\left(-\frac{1}{2}, \frac{\sqrt{3}}{2}, 0\right) \\
& \mathbf{n}_{3}=\left(-\frac{1}{2},-\frac{\sqrt{3}}{2}, 0\right)
\end{aligned}
$$


a direct substitution of (3.21) into (3.14) gives

$$
\frac{\partial \mathbf{n}_{i}}{\partial t}=-\boldsymbol{\lambda}_{\mathrm{cr}} \times \mathbf{n}_{i}
$$

where $\boldsymbol{\lambda}_{\mathrm{cr}}$ coincides with the symmetry axis of the triangle,

$$
\lambda_{\mathrm{cr}}=\frac{\sqrt{3}}{2}(0,0, c)
$$

Thus, whenever $c \neq 0$ we have a time crystal that describes an equilateral triangle that rotates around its symmetry axis with an angular velocity that is linearly proportional to the parameter $c$ in (3.21). Note that for $c=0$ only the first term in (3.21) is present. For this $c$-value $\boldsymbol{\lambda}$ vanishes; there is no time crystal if only $H_{1}(\mathbf{n})$ is present.

The present example is a very simple realization of the general result (2.12), as the equilateral triangle can not change its shape and the Hamiltonian (3.21) has only a single value which is simultaneously the minimum and maximum of the available energy.

\subsection{Four-vertex model}

The Hamiltonian (3.21) can be readily extended to a closed string with more than $N=3$ variables. As an example we consider a closed polygonal string with $N=4$ vertices, and for convenience we take $\left|\mathbf{x}_{i+1}-\mathbf{x}_{i}\right|=1$. Geometrically, we may view the $\mathbf{x}_{i}$ as the vertices of a tetrahedron in $\mathbb{R}^{3}$ with four equal length edges. Up to an additive constant the Hamiltonian (3.21) is

$$
H=H_{1}+H_{2}=-\sum_{i=1}^{4}\left(\mathbf{n}_{i} \cdot \mathbf{n}_{i+1}\right)^{2}+c \mathbf{n}_{1} \cdot\left(\mathbf{n}_{2} \times \mathbf{n}_{3}\right) \quad\left(\text { with } \mathbf{n}_{5}=\mathbf{n}_{1}\right)
$$

To construct a time crystal, we first minimize the energy (3.22) on the constraint manifold (3.15) for different parameter values $c$. Since the four edges have equal length, $H$ is a function of the following two conformational angles,

$$
\begin{array}{ll}
\alpha=\pi-\arccos \left(\mathbf{n}_{1} \cdot \mathbf{n}_{4}\right) & \in(0, \pi] \\
\beta=\pi-\arccos \left(\frac{\left(\mathbf{n}_{1}-\mathbf{n}_{4}\right) \cdot\left(\mathbf{n}_{3}-\mathbf{n}_{2}\right)}{\left|\mathbf{n}_{1}-\mathbf{n}_{4}\right|\left|\mathbf{n}_{3}-\mathbf{n}_{2}\right|}\right) & \in(0, \pi]
\end{array}
$$

Here $\alpha$ is the bond angle $\angle\left(\mathbf{x}_{4} \mathbf{x}_{1} \mathbf{x}_{2}\right)$ and $\beta$ is the dihedral angle between the two planes $\left.<\mathbf{x}_{1} \mathbf{x}_{2} \mathbf{x}_{4}\right\rangle$ and $\left.<\mathbf{x}_{2} \mathbf{x}_{3} \mathbf{x}_{4}\right\rangle$. Accordingly, we proceed and express the Hamiltonian (3.22) in terms of (3.23), and then search for the minimum of $H(\alpha, \beta)$ with different parameter values $c$. For energy minimization we use a two dimensional adaptive grid algorithm.

We find that there is a critical value $c=4.0$ with the following properties.

When $c<4.0$ the minimum of $H(\alpha, \beta)$ is doubly degenerate, both $(\alpha, \beta)=(0,0)$ and $(\alpha, \beta)=(0, \pi)$ are minima of the energy and at these minima we have $H=H_{1}=-4$; $H_{2}=0$. Geometrically, in both energy minima the four link vectors $\mathbf{n}_{i}$ are all aligned with each other in $\mathbb{R}^{3}$. In the case of $(\alpha, \beta)=(0,0)$ the neighboring vectors $\mathbf{n}_{1}$ and $\mathbf{n}_{2}$ are mutually parallel and opposite to the mutually parallel $\mathbf{n}_{3}$ and $\mathbf{n}_{4}$. In the case of $(\alpha, \beta)$ 

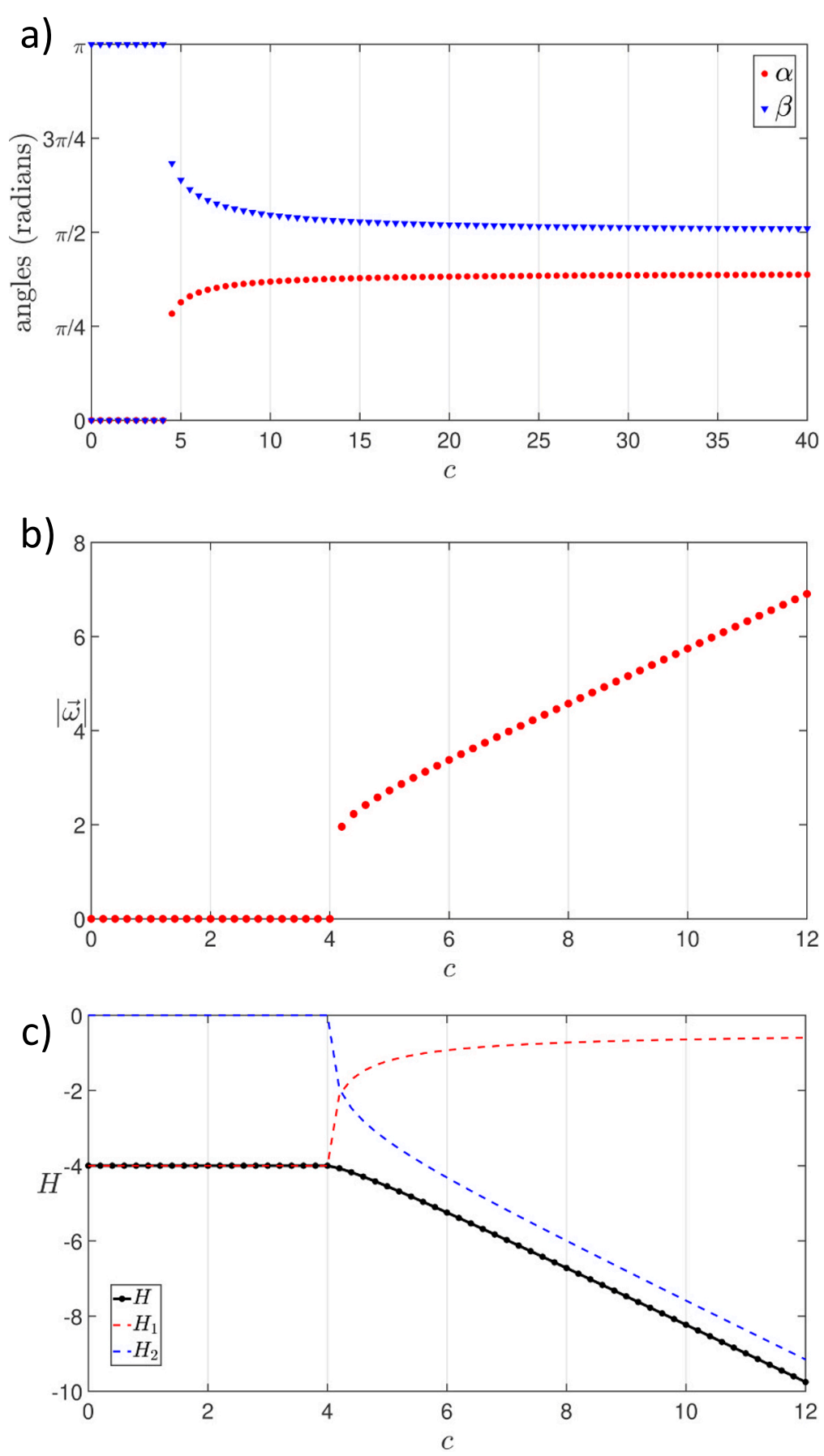

Figure 1. Panel a) The minimum energy values of the angles (3.23) as a function of the parameter $c$ in (3.22). The asymptotic large- $c$ values $(\alpha, \beta)=(\arccos (1 / 3), \pi / 2)$ correspond to a tetragonal disphenoid.Panel b) The angular velocity of the time crystal with Hamiltonian (3.22) as a function of the parameter $c$. Panel c) The total energy $H$ and the individual contributions $H_{1}$ and $H_{2}$ in (3.22), as a function of parameter $c$ for the time crystal. 
$=(0, \pi)$ the vectors $\mathbf{n}_{1}$ and $\mathbf{n}_{3}$ are mutually parallel, and opposite to the direction of the mutually parallel $\mathbf{n}_{2}$ and $\mathbf{n}_{4}$. When we substitute in (3.14) we find no motion, neither of the two energy minima corresponds to a time crystal and we conclude that for $c<4.0$ there is no time crystal.

When $c=4.0$ we observe an abrupt change in the values of $(\alpha, \beta)$. Figure 1 Panel a) shows how the values of $(\alpha, \beta)$ jump from the $c<4.0$ minimum energy values $(\alpha, \beta)=(0,0)$, $(0, \pi)$ to $(\alpha, \beta) \approx(0.87,2.18)$ radians for $c=4.0+$, and Panel $\mathrm{b})$ shows how the angular velocity also changes abruptly. We observe a change in the shape of the minimum energy polygonal string, for $c<4.0$ the distances $d_{13}=\left|\mathbf{x}_{3}-\mathbf{x}_{1}\right|$ and $d_{24}=\left|\mathbf{x}_{4}-\mathbf{x}_{2}\right|$ have the values $d_{13}=0$ and $d_{24}=2$, but for $c=4.0+$ these values are $d_{13}=d_{24}=0.8$.

When $c>4.0$ we find that the minimum energy configuration is a time crystal, it rotates with an angular velocity $|\boldsymbol{\omega}|$ that increases when $c$ increases; see figure 1 Panel b). At the same time, the total energy decreases as a function of $c$, as shown in figure 1 Panel c). Asymptotically, for large values of $c$, both the minimum value of the energy and the angular velocity depend on $c$ linearly. For the conformational angles the large$c$ limiting values are $(\alpha, \beta)=(\arccos (1 / 3), \pi / 2)$ and thus, in the large- $c$ limit the four vertices $\mathbf{x}_{1}, \ldots, \mathbf{x}_{4}$ approach the vertices of the space filling tetragonal disphenoid with $d_{13}=d_{24}=2 / \sqrt{3} ;$ see figure 1 Panel a).

\section{Example 2: time crystals from a reduction of the Q-ball Lagrangian}

Our second example builds on the Q-ball model of [34]; for surveys see [35, 36]. There is one complex field $\varphi(\mathbf{x}, t)$ and the Hamiltonian form of the (relativistic) action is

$$
\int d t d^{D} x\left\{\pi \dot{\varphi}+\pi^{\star} \dot{\varphi}^{\star}-\pi \pi^{\star}-|\nabla \varphi|^{2}-U(|\varphi|)\right\}=\int d t d^{D} x\left\{\pi \dot{\varphi}+\pi^{\star} \dot{\varphi}^{\star}-H(\pi, \varphi)\right\}
$$

The non-vanishing Poisson brackets are

$$
\{\varphi(\mathbf{x}), \pi(\mathbf{y})\}=\left\{\varphi^{\star}(\mathbf{x}), \pi^{\star}(\mathbf{y})\right\}=\delta(\mathbf{x}-\mathbf{y})
$$

There is also a conserved charge

$$
\begin{aligned}
Q & =i \int d^{D} x\left(\pi \varphi-\pi^{\star} \varphi^{\star}\right) \\
\frac{d Q}{d t} & =\{Q, H\}=0
\end{aligned}
$$

and we conclude from Hamilton's equations

$$
\begin{aligned}
& \dot{\varphi}=\{\varphi, H\}=\pi \\
& \dot{\pi}=\{\pi, H\}=\nabla^{2} \varphi-\frac{\partial U}{\partial \varphi}
\end{aligned}
$$

that whenever the charge (4.2) has a non-vanishing value $q$, the field $\varphi$ must vary with time. But not all $q \neq 0$ minimum energy configurations are Q-balls. For (4.1), (4.2) to support a Q-ball the potential $U(\varphi)$ needs to be chosen in a particular fashion. 


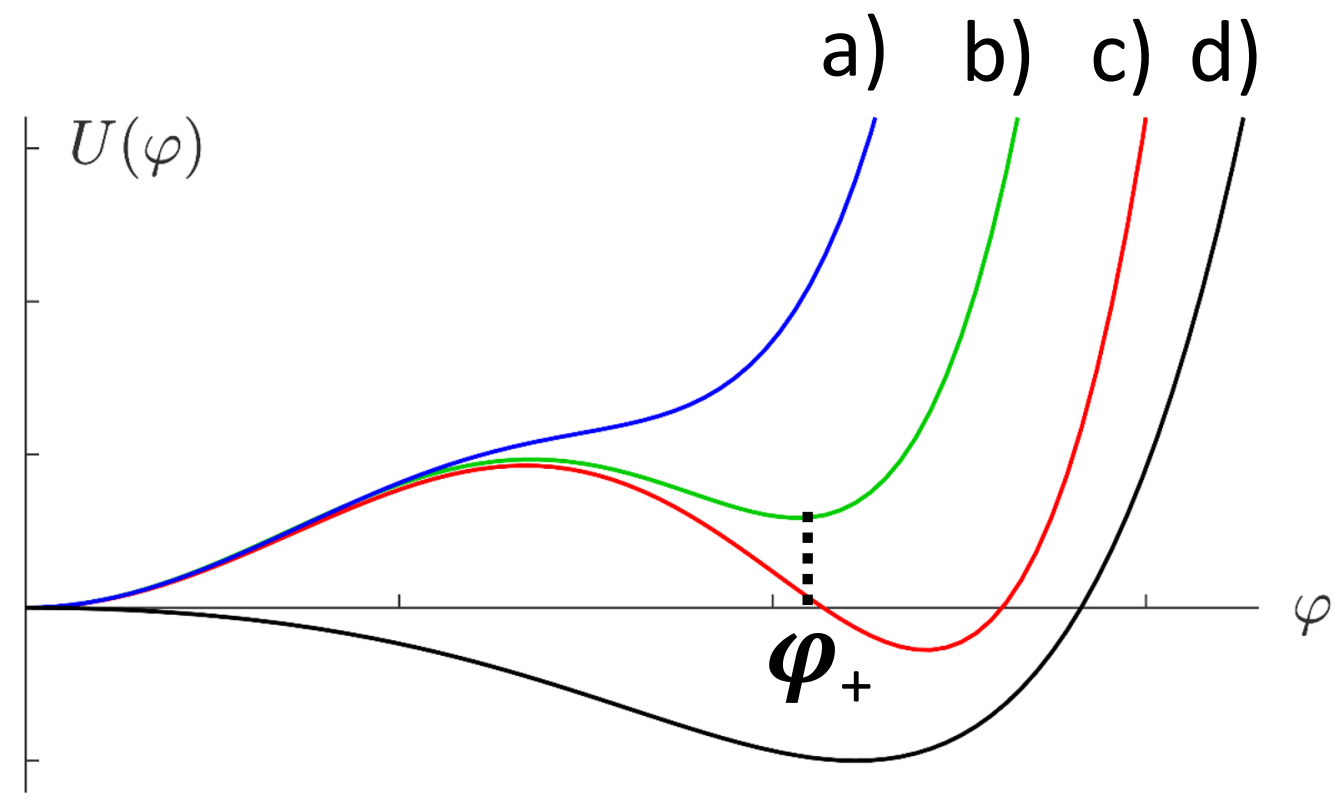

Figure 2. The potential a) has a single global minimum, at the origin. The potential b) has a global minimum at origin, and an additional local minimum at non-vanishing value $\varphi_{+}$. The potential c) has a local minimum at the origin, and a global minimum at a non-vanishing value of $\varphi$. The potential d) has a local maximum at the origin, and a global minimum at a non-vanishing value of $\varphi$. As shown in [34] a potential with the profile b) can support Q-balls in $D=3$

In [34] a Q-ball potential with the following properties was introduced, in the case of $D=3$. There should be no spontaneous symmetry breaking, the origin $\varphi_{0}=0$ should be the global minimum of $U(\varphi)$. In addition $U(\varphi)$ should have a local minimum at $\varphi_{+} \neq 0$ and in the limit $|\varphi| \rightarrow \infty$ the value of $U(\varphi)$ should go to infinity. Accordingly, the profile of $U(\varphi)$ should resemble the potential b) in figure 2. A Q-ball can then form, when for small $|\mathbf{x}|$ the field $\varphi(\mathbf{x}, t)$ acquires a value close to $\varphi_{+}$and approaches the global minimum $\varphi_{0}=0$ of $U(\varphi)$ for large $|\mathbf{x}|$. The Q-ball is a stable finite energy spherical domain wall that separates the two ground states, it is a non-topological soliton that interpolates between the ground state $\varphi_{+}$in its interior and the ground state $\varphi_{0}$ in its exterior, with a profile and time dependence specified by the details of the action.

We inquire whether a Q-ball can be related to a time crystal: a timecrystalline Q-ball would be a time dependent minimum of the Hamiltonian energy in (4.1) with charge $q \neq 0$. We search for such an energy minimum using the general formalism of section 2 .

From (4.1), (4.2) the Hamiltonian (2.10) is

$$
H_{\lambda}=\int d^{D} x\left\{\pi \pi^{\star}+|\nabla \varphi|^{2}+|\varphi|^{2}-|\varphi|^{4}+\mu|\varphi|^{6}\right\}+i \lambda\left\{\int d^{D} x\left(\pi \varphi-\pi^{\star} \varphi^{\star}\right)+i q\right\}
$$

where the Lagrange multiplier $\lambda$ enforces the conserved charge (4.2) to have the prescribed value $q \neq 0$. When $\pi_{\mathrm{cr}}, \varphi_{\mathrm{cr}}, \lambda_{\mathrm{cr}}$ solve the pertinent equation (2.11) so that $\pi_{\mathrm{cr}}, \varphi_{\mathrm{cr}}$ minimize the energy in (4.1) with a domain wall profile, akin a Q-ball, and $\lambda_{\text {cr }} \neq 0$, we have a 
timecrystalline Q-ball with time evolution given by (2.12),

$$
\begin{array}{lll}
\dot{\varphi}=i \lambda_{\mathrm{cr}} \varphi & \text { with } & \varphi(0, \mathbf{x})=\varphi_{\mathrm{cr}}(\mathbf{x}) \\
\dot{\pi}=-i \lambda_{\mathrm{cr}} \pi & \text { with } & \pi(0, \mathbf{x})=\pi_{\mathrm{cr}}(\mathbf{x})
\end{array}
$$

For a numerical construction of a domain wall that makes a timecrystalline Q-ball, we consider a discrete variant of (4.5). We set $D=1$ for computational simplicity, so that we have a one dimensional lattice with $N$ sites. Thus we search for a timecrystalline domain wall along a one dimensional lattice chain: we discretize the gradient and we redefine (rescale) the variables so that we are left with the following version of (2.10)

$$
\begin{aligned}
H_{\lambda}= & H+\lambda(Q-q) \\
= & \sum_{k=1}^{N}\left\{\pi_{k} \pi_{k}^{\star}-\epsilon\left(\varphi_{k+1}^{\star} \varphi_{k}+\varphi_{k+1} \varphi_{k}^{\star}\right)+\left|\varphi_{k}\right|^{2}-\left|\varphi_{k}\right|^{4}+\mu\left|\varphi_{k}\right|^{6}\right\} \\
& +i \lambda\left\{\sum_{k=1}^{N}\left(\pi_{k} \varphi_{k}-\pi_{k}^{\star} \varphi_{k}^{\star}\right)+i q\right\}
\end{aligned}
$$

where the second term emerges from the cross-term of the discretized gradient; we set $\varphi_{N+1}=0$.

In a conceivable physical application, the values $(\epsilon, \mu)$ characterize a particular physical environment and the values $q$ of the conserved charge specifies the presymplectic slice $\mathcal{M}_{g}$ in (2.6). Accordingly, we search for timecrystalline Q-ball domain walls, characterized by the value of $q$ in the environment of parameter values $(\epsilon, \mu)$.

The critical points $\left(\varphi_{k, c r}, \pi_{k, c r}, \lambda_{\mathrm{cr}}\right)$ of (4.7) are solutions to the ensuing equations (2.11),

$$
\begin{aligned}
\pi_{k} & =i \lambda \varphi_{k}^{\star} \\
\epsilon\left(\varphi_{k-1}+\varphi_{k+1}\right)-\varphi_{k}+2\left|\varphi_{k}\right|^{2} \varphi_{k}-3 \mu\left|\varphi_{k}\right|^{4} \varphi_{k} & =-i \lambda \pi_{k}^{\star} \quad\left(\varphi_{0}=0\right) \\
\pi_{k} \varphi_{k}-\pi_{k}^{\star} \varphi_{k}^{\star} & =-i q
\end{aligned}
$$

and we follow the steps of section 2 to numerically search for a timecrystalline solution: for a time crystal we need to find a choose the solution of (4.8) that corresponds to the global minimum of the energy

$$
\min _{\pi_{k}, \varphi_{k} \in \mathbb{R}}\left\{\sum_{k=1}^{N} \pi_{k} \pi_{k}^{\star}-\epsilon\left(\varphi_{k+1}^{\star} \varphi_{k}+\varphi_{k+1} \varphi_{k}^{\star}\right)+\left|\varphi_{k}\right|^{2}-\left|\varphi_{k}\right|^{4}+\mu\left|\varphi_{k}\right|^{6}\right\}
$$

subject to the condition

$$
\sum_{k=1}^{N}\left(\pi_{k} \varphi_{k}-\pi_{k}^{\star} \varphi_{k}^{\star}\right)=-i q
$$

The solution of (4.8) is not unique, the landscape of solutions is quite complex. It becomes very tedious to ensure that we have indeed located the solution that yields the global minimum of (4.9), (4.10). Thus, instead of solving (4.8) we directly search for 
a minimum of (4.9), (4.10). For this we adopt the constrained nonlinear optimization algorithm developed in [37] that we implement using $M A T L A B$. The algorithm provides us with a set of critical values $\pi_{k, c r}$ and $\varphi_{k, c r}$ and we select those that correspond to a global, not just a local, minimum of energy (4.9). We ensure that we have a global minimum, by using a large pool of randomly generated initial values in the optimization algorithm [37]. Once the minimum of the energy has been localized, we evaluate $\lambda_{\mathrm{cr}}$ from the first equation (4.8),

$$
\lambda_{\mathrm{cr}}=i \frac{\pi_{k, c r}^{\star}}{\varphi_{k, c r}}
$$

For a time crystal $\lambda_{\text {cr }} \neq 0$ and in particular we verify that $\lambda_{\text {cr }}$ has a value which is independent of the index $k$ which serves as a consistency check of our minimization result.

Even constrained nonlinear optimization is numerically very tedious, as the complexity of the energy landscape grows rapidly with the number of variables $N$. Thus the computer time we need to ensure that we have a global and not just a local energy minimum, increases very quickly when $N$ increases. For this reason we have limited our search of timecrystalline Q-ball to a relatively small number of lattice sites $N$.

As an example, in the figures 3 and 4 we sketch out our results in the simplest nontrivial case, with $N=5$ lattice sites (and with $\epsilon=1 / 8$ ); we have found that for values larger than $N=5$ the results are qualitatively very similar. We select $\mu \in[0.15,2.5]$ and the conserved charge has values in the range $q \in[0,4]$, as we have found that in this range of $(\mu, q)$ values timecrystalline Q-ball domain walls can be found. For numerical simulation, we divide $[\mu, q]$ into a 100x100 lattice and for each pair $(\mu, q)$ we perform 100 independent minimizations, starting from randomly chosen initial values. In the figures 3 and 4 we use

$$
\rho_{k}=\sqrt{\varphi_{k, c r}^{\star} \varphi_{k, c r}} \quad k=1, \ldots, 5
$$

as the order parameter. For the corresponding momentum order parameters

$$
\sigma_{k}=\sqrt{\pi_{k, c r}^{\star} \pi_{k, c r}} \quad k=1, \ldots, 5
$$

the results are very similar since according to (4.11) $\sigma_{k} / \rho_{k}=\left|\lambda_{\text {cr }}\right|$ for all $k$.

Figure 3 panels I-VI show examples of the energy minima that we find, for different parameter values. The six examples we depict are generic and chosen to describe what we find in the range of parameters we investigate. The panels are ordered according to increasing conserved charge value $q$.

- The Panel I characterizes the small- $q$ and large- $\mu$ region. We propose that this configuration corresponds to a situation akin that shown in figure 2 profile a): in terms of such an effective potential energy interpretation, there is only the global minimum that is located at $\rho \approx 0$ (i.e. very small). In particular, there is no domain wall.

- The Panel II shows how the minimum energy configuration in Panel I evolves when we increase the value of $q$, and lower the value of $\mu$ : the value of $\rho_{3}$ at the center of the chain increases until it reaches $\rho_{3} \approx 0.7$ as shown in the Panel II. In terms of 

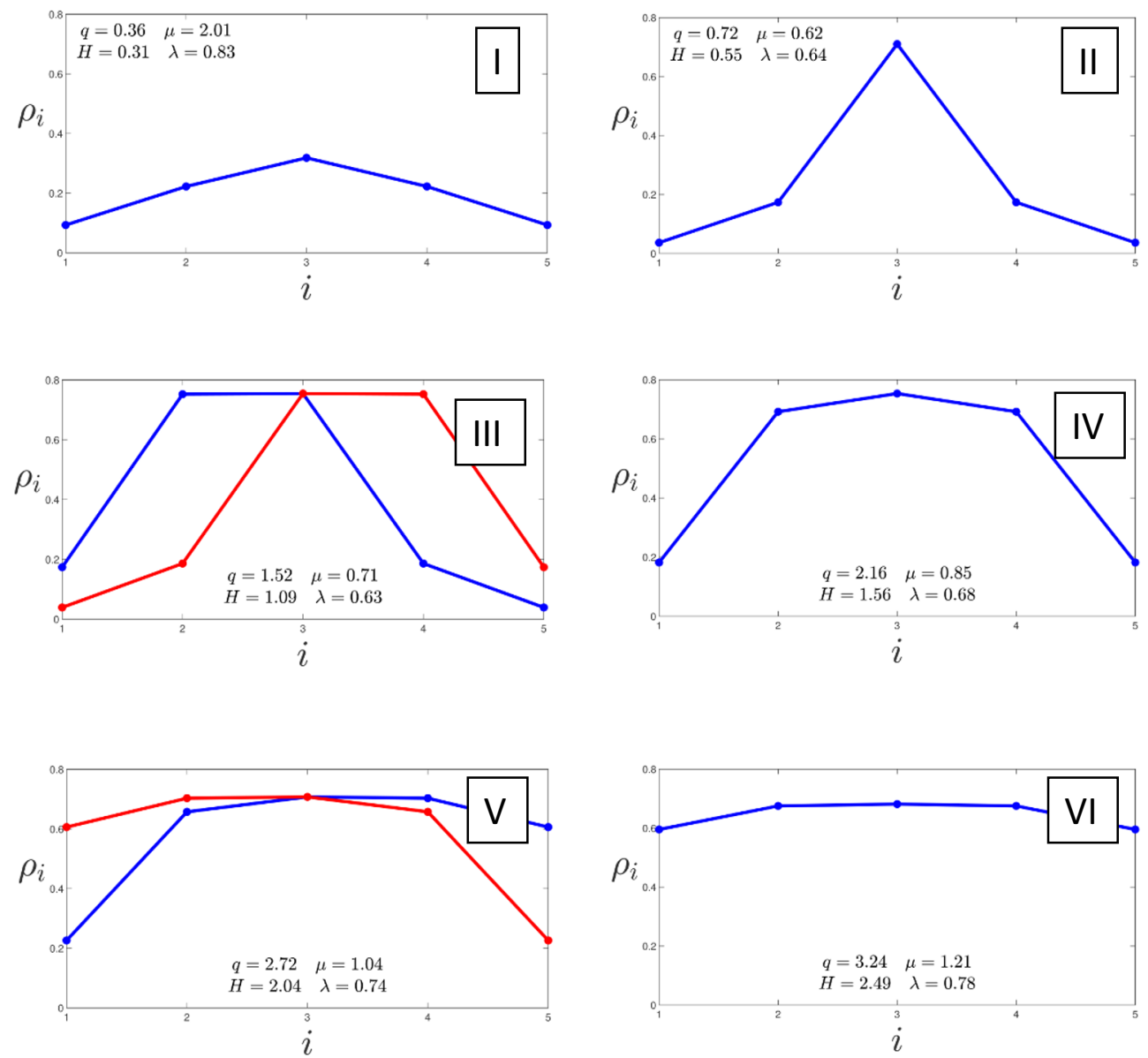

Figure 3. Values of order parameter (4.12) for minimum energy configuration (4.9), (4.10) with different representative parameter values $(q, \mu)$ for a chain with $N=5$ sites and with $\epsilon=1 / 8$. Panels I and VI do not describe a timecrystalline Q-ball; there is no domain wall. Panels II-IV are timecrystalline Q-balls with two domain walls; note that the blue and red profiles in Panel III are reflection symmetric under $k \rightarrow 6-k$. In Panel $\mathrm{V}$ we have a pair of $k \rightarrow 6-k$ reflection symmetric timecrystalline single domain wall Q-balls.

figure 2 we propose the interpretation that the effective potential energy approaches a transition between the profiles a) and b). That is, a local minimum emerges in the effective potential energy, near $\rho \approx 0.7$ : the profile resembles a pair of domain walls, close to each other.

- In Panels III and IV we increase the value of $q$, with only small changes in $\mu$. In both Panels the $\rho$ values move back and forth between $\rho \approx 0$ and $\rho \approx 0.7$, when we move along the chain. Note that in Panel III the energy minimum has a two-fold $k \rightarrow 6-k$ degeneracy but in Panel IV the energy minimum is $k \rightarrow 6-k$ symmetric. In terms of the effective potential energy description of figure 2 we propose that these Panels correspond to the case b) with only a small energy difference between the two ground states: the profiles describe two domain walls that move away from each other as $q$ increases. 
- In the Panel V we increase the value of $q$ further while the value of $\mu$ is more or less intact. The energy minimum interpolates between $\rho \approx 0$ and $\rho \approx 0.7$ and we again observe a two-fold $k \rightarrow 6-k$ degeneracy. In terms of the effective potential energy description of figure 2 we propose that this Panel corresponds to the case between $b$ ) and c) with the qualitative feature of the Q-ball potential energy of [34-36]: each of the degenerate energy minima describes a single domain wall.

- Finally, Panel VI represents the region of large $q$ but not so small $\mu$ values. Now $\rho$ appear to reside in the ground state with $\rho \approx 0.7$. The energy minimum resembles the scenario d) of figure 2 and there is no domain wall.

The figure 4 Panels a)-e) show the entire $(q, \mu)$-landscape of $\rho_{k}$ minimum energy solutions, in the range of $(q, \mu)$ values that we have studied; the Panels show the landscape of minimum energy $\rho_{k}$ values both as a surface map and as a contour map. In each of the individual Panels we have marked the six solutions that we have detailed in figures 3.

The Panel $\mathrm{f}$ ) of the figure 4 shows the values of $\lambda_{\mathrm{cr}}(q, \mu)$, evaluated from (4.11). The Panel shows that each of the energy minima in figures 3 have $\lambda_{\text {cr }} \neq 0$. Thus they are all time dependent energy minima. Each determines a time dependent, timecrystalline symmetry transformation (2.12) of the Hamiltonian, of the form

$$
\begin{aligned}
& \varphi_{k}(t)=\varphi_{k, c r}(\mu, q) e^{i \lambda_{\mathrm{cr}}(\mu, q) t} \\
& \pi_{k}(t)=\pi_{k, c r}(\mu, q) e^{-i \lambda_{\mathrm{cr}}(\mu, q) t}
\end{aligned}
$$

For each lattice site $k$ this describes uniform rotation on the $\left(\varphi_{k}, \pi_{k}\right)$ plane with angular velocity $\lambda_{\text {cr }}(\mu, q)$ independently of the $k$-value.

From figures 3 and 4 we confirm that the energy minima shown in Panels I and VI of figure 3 have no domain walls. Thus they are not Q-ball domain walls, in the sense described in [34]. Accordingly we do not consider them to be time crystals, either: these two Panels describe minimum energy configurations with (essentially) uniform $\rho_{k}$-values, there is no interpolation between different ground states as there is no domain wall. Thus we can smoothly deform them to the ensuing ground state, with $q=0$. From the point of view of our general formalism in section 2, in these two cases the corresponding orbit $\mathcal{M}_{g}$ is path connected to a time independent minimum energy configuration with $q=0$. Moreover, figures 4 show that we can path connect these two energy minima to each other, continuously in the $(q, \mu)$ plane, without encountering any domain wall region in between, by a uniform increase/decrease of the $\rho_{k}$ values.

The situation is different in the cases shown in Panels II-V of figure 3. In each of these Panels, the minimum energy configuration has a domain wall profile. Accordingly, in line with [34] we interpret these four cases of energy minima as genuine timecrystalline Q-ball domain walls. Note that the example in Panel V is a time crystalline Q-ball, with a single domain wall, in line with the Q-ball constructed in [34]. The examples in Panels III and IV display time crystalline Q-ball domain wall pairs, the two domain walls move and come together as shown in Panel II, when $q$ decreases. We remark that both spherical and toroidal Q-balls have been described in the literature [36]. 
a)

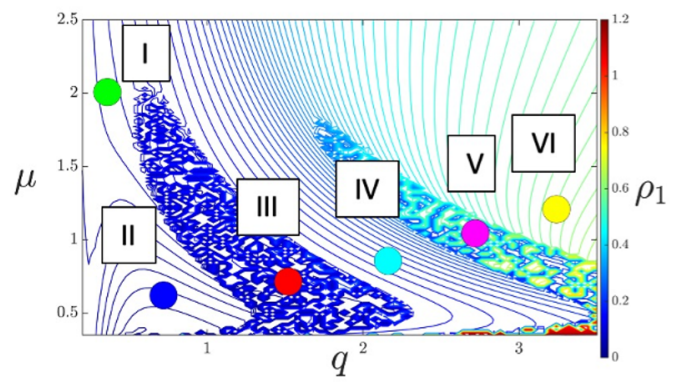

c)

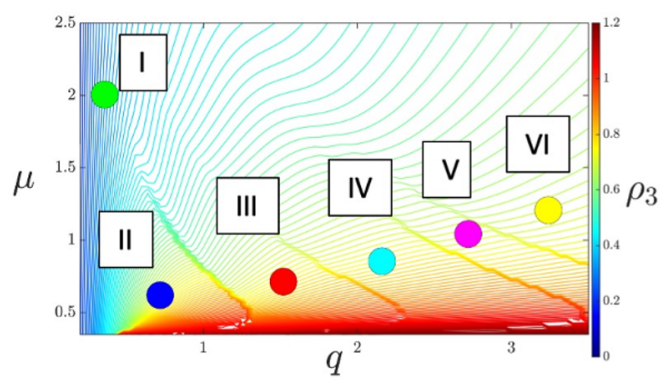

e)

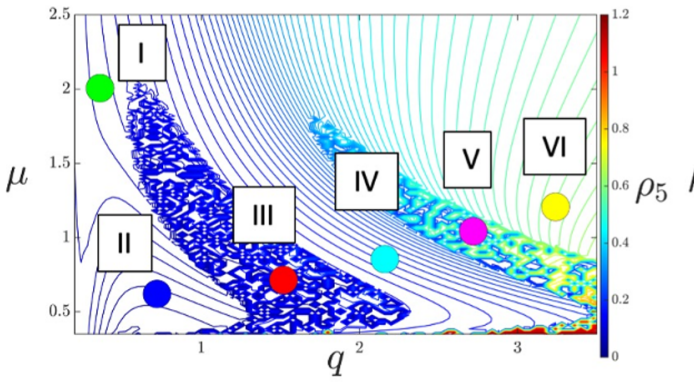

b)

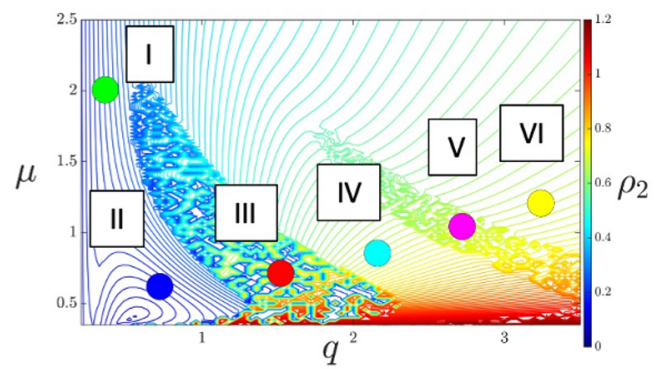

d)

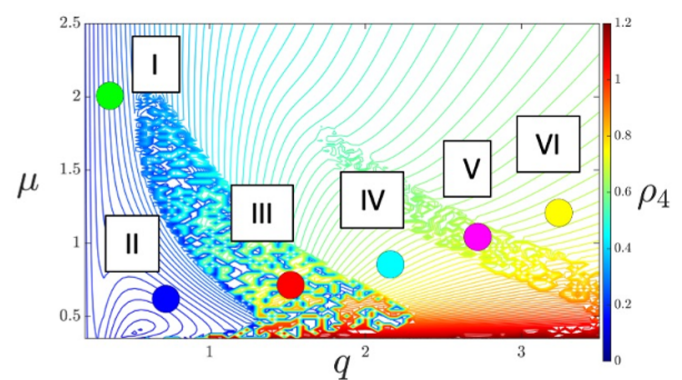

f)

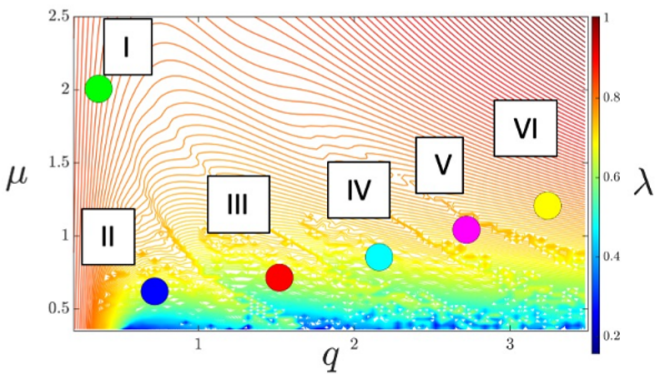

Figure 4. The landscape of timecrystalline Q-balls in terms of $(q, \mu)$. The Panels a)-e) show the landscape for the five order parameters $\rho_{1} \ldots \rho_{5}$, respectively. In each panel the $\rho_{k}$ of the six energy minima shown in figures 3 are identified. The roughness of the landscape is due to a $k \rightarrow 6-k$ symmetry: a timecrystalline Q-ball corresponds to a double degenerate ground state energy and the roughness describes how at each point the landscape is constructed from a randomly chosen initial configuration, to display the presence of double degeneracy. Panel f) then shows the $\left|\lambda_{\mathrm{cr}}\right|$ values, evaluated from (4.11).

\section{Conclusions}

We have shown, both using the general formalism of geometric mechanics and in terms of explicit examples, that classical Hamiltonian time crystals do exist and can be found in Hamiltonian systems with conserved charges. We have presented two general examples, the first describes timecrystalline discrete closed strings and the second describes timecrystalline Q-ball domain walls. 
The No-Go arguments only apply on a phase space with a symplectic structure, but in the case of a time crystal the phase space is pre-symplectic. In particular, our general formalism establishes that a symmetry is necessary for the existence of a time crystal. Thus the provenance of a Hamiltonian time crystal lies in the general phenomenon of spontaneous symmetry breaking that now takes place in a dynamical context.

\section{Acknowledgments}

The research of AA was supported in part by the National Center for Competence in Research (NCCR) SwissMAP and by the grants number 178794 and 178828 of the Swiss National Science Foundation. AA is grateful for hospitality to the CRM of the University of Montreal and to the IHES where this project was developed. The work by DJ and AJN is supported by the Carl Trygger Foundation and by the Swedish Research Council (VR), DJ and AJN also acknowledge collaboration under COST Action CA17139. DJ and AJN thank Frank Wilczek and Xubiao Peng for discussions.

Open Access. This article is distributed under the terms of the Creative Commons Attribution License (CC-BY 4.0), which permits any use, distribution and reproduction in any medium, provided the original author(s) and source are credited.

\section{References}

[1] F. Wilczek, Quantum time crystals, Phys. Rev. Lett. 109 (2012) 160401 [arXiv:1202.2539] [INSPIRE].

[2] A. Shapere and F. Wilczek, Classical time crystals, Phys. Rev. Lett. 109 (2012) 160402 [arXiv: 1202.2537] [INSPIRE].

[3] F. Wilczek, Superfluidity and space-time translation symmetry breaking, Phys. Rev. Lett. 111 (2013) 250402.

[4] A. Shapere and F. Wilczek, Regularizations of time-crystal dynamics, PNAS 116 (2019) 18772 .

[5] K. Sacha and J. Zakrzewski, Time crystals: a review, Rep. Prog. Phys. 81 (2018) 016401 [arXiv:1704.03735].

[6] N.Y. Yao and C. Nayak, Time crystals in periodically driven systems, Phys. Today 71 (2018) 40.

[7] K. Sacha, Modeling spontaneous breaking of time-translation symmetry, Phys. Rev. A 91 (2015) 033617 [arXiv:1410.3638] [INSPIRE].

[8] V. Khemani, A. Lazarides, R. Moessner and S.L. Sondhi, Phase structure of driven quantum systems, Phys. Rev. Lett. 116 (2016) 250401.

[9] D.V. Else and C. Nayak, Classification of topological phases in periodically driven interacting systems, Phys. Rev. B 93 (2016) 201103.

[10] D.V. Else, B. Bauer and C. Nayak, Floquet time crystals, Phys. Rev. Lett. 117 (2016) 090402.

[11] D.V. Else, B. Bauer and C. Nayak, Prethermal phases of matter protected by time-translation symmetry, Phys. Rev. X 7 (2017) 011026 [arXiv:1607.05277] [INSPIRE]. 
[12] N.Y. Yao, A.C. Potter, I.-D. Potirniche and A. Vishwanath, Discrete time crystals: rigidity, criticality, and realizations, Phys. Rev. Lett. 118 (2017) 030401 [Erratum ibid. 118 (2017) 269901].

[13] D.V. Else, C. Monroe, C. Nayak and N.Y. Yao, Discrete time crystals, arXiv:1905.13232.

[14] J. Zhang et al., Observation of a discrete time crystal, Nature 543 (2017) 217.

[15] S. Choi et al., Dynamics of interacting fermions under spin-orbit coupling in an optical lattice clock, Nature Phys. 543 (2017) 221.

[16] S. Pal, N. Nishad, T.S. Mahesh and G.J. Sreejith, Temporal order in periodically driven spins in star-shaped clusters, Phys. Rev. Lett. 120 (2018) 180602.

[17] J. Rovny, R.L. Blum, S.E. Barrett, Observation of discrete-time-crystal signatures in an ordered dipolar many-body system, Phys. Rev. Lett. 120 (2018) 180603.

[18] J. Rovny, R.L. Blum and S.E. Barrett, ${ }^{31} P$ NMR study of discrete time-crystalline signatures in an ordered crystal of ammonium dihydrogen phosphate, Phys. Rev. B 97 (2018) 184301.

[19] J. Smits, L. Liao, H.T.C. Stoof and P. van der Straten, Observation of a space-time crystal in a superfluid quantum gas, Phys. Rev. Lett. 121 (2018) 185301.

[20] J. Smits, L. Liao, H.T.C. Stoof and P. van der Straten, Dynamics of a space-time crystal in an atomic Bose-Einstein condensate, Phys. Rev. A 99 (2018) 013625.

[21] K. Giergiel, A. Kosior, P. Hannaford and K. Sacha, Time crystals: analysis of experimental conditions, Phys. Rev. A 98 (2018) 013613.

[22] P. Bruno, Comment on "Quantum time crystals", Phys. Rev. Lett. 110 (2013) 118901 [arXiv: 1210.4128] [INSPIRE].

[23] H. Watanabe and M. Oshikawa, Absence of quantum time crystals, Phys. Rev. Lett. 114 (2015) 251603 [arXiv: 1410.2143] [INSPIRE].

[24] J. Dai, A.J. Niemi, X. Peng and F. Wilczek, Truncated dynamics, ring molecules, and mechanical time crystals, Phys. Rev. A 99 (2019) 023425.

[25] J.E. Marsden and T.S. Ratiu, Introduction to mechanics and symmetry a basic exposition of classical mechanical systems, second Edition, Springer, Germany (1999).

[26] P.A.M. Dirac, Lectures on quantum mechanics, Belfer Graduate School of Science Monographs Series volume 2, New York, U.S.A. (1964).

[27] A. Wasserman, Equivariant differential topology, Topology 8 (1969) 127.

[28] A.J. Niemi and K. Palo, Equivariant Morse theory and quantum integrability, hep-th/9406068 [INSPIRE].

[29] D.M. Austin and P.J. Braam, Morse-Bott theory and equivariant cohomology in The Floer memorial volume, H. Hofer et al. eds., Progress in Mathematics volume 133, Birkhäuser, Basel Switzerland (1995).

[30] L. Nicolaescu, An invitation to morse theory, second edition, Springer, Germany (2011).

[31] A.Y. Alekseev, A. Recknagel and V. Schomerus, Brane dynamics in background fluxes and noncommutative geometry, JHEP 05 (2000) 010 [hep-th/0003187] [INSPIRE].

[32] S. Monnier, D-branes in Lie groups of rank \& 1, JHEP 08 (2005) 062 [hep-th/0507159] [INSPIRE]. 
[33] A. Alekseev and F. Petrov, A principle of variations in representation theory, in The orbit method in geometry and physics: in honor of A.A. Kirillov, C. Duval et al. eds., Progress in Mathematics volume 213, Birkhäuser, Boston U.S.A. (2000).

[34] S.R. Coleman, Q balls, Nucl. Phys. B 262 (1985) 263 [Erratum ibid. 269 (1986) 744] [INSPIRE].

[35] T.D. Lee and Y. Pang, Nontopological solitons, Phys. Rept, 221 (1992) 251.

[36] E. Radu and M.S. Volkov, Existence of stationary, non-radiating ring solitons in field theory: knots and vortons, Phys. Rept. 468 (2008) 101 [arXiv:0804.1357] [INSPIRE].

[37] R.H. Byrd, J.C. Gilbert and J. Nocedal, A trust region method based on interior point techniques for nonlinear programming, Math. Progr. 89 (2000) 149. 